\title{
Image Segmentation of Mesenchymal Stem Cells in Diverse Culturing Conditions*
}

\author{
Muhammad Jamal Afridi ${ }^{1}$, Chun $\mathrm{Liu}^{2}$, Christina Chan $^{2}$, Seungik Baek ${ }^{3}$, and Xiaoming Liu ${ }^{\dagger}$ \\ ${ }^{1}$ Department of Computer Science and Engineering, Michigan State University \\ ${ }^{2}$ Department of Biochemistry and Molecular Biology, Michigan State University \\ ${ }^{3}$ Department of Mechanical Engineering, Michigan State University \\ 1,2,3 \{afridimu,liuchun2,krischan,sbaek,liuxm\}@msu.edu
}

\begin{abstract}
Researchers in the areas of regenerative medicine and tissue engineering have great interests in understanding the relationship of different sets of culturing conditions and applied mechanical stimuli to the behavior of mesenchymal stem cells (MSCs). However, it is challenging to design a tool to perform automatic cell image analysis due to the diverse morphologies of MSCs. Therefore, as a primary step towards developing the tool, we propose a novel approach for accurate cell image segmentation. We collected three MSC datasets cultured on different surfaces and exposed to diverse mechanical stimuli. By analyzing existing approaches on our data, we choose to substantially extend binarization-based extraction of alignment score (BEAS) approach by extracting novel discriminating features and developing an adaptive threshold estimation model. Experimental results on our data shows our approach is superior to seven conventional techniques. We also define three quantitative measures to analyze the characteristics of images in our datasets. To the best of our knowledge, this is the first study that applied automatic segmentation to live MSC cultured on different surfaces with applied stimuli.
\end{abstract}

\section{Introduction}

In 2006, about 6,300 people died in U.S. because of vital organ failure [3]. According to the Department of Health and Human Services, about 18 people die in U.S. everyday while waiting for an organ transplant [1]. To remedy this issue, scientists have seen a promising solution for organ transplant rejection, organ failure, and cardiovascular repair by using regenerative medicine [2]. Hence, studies related to understanding the behavior of mesenchymal stem cells

${ }^{*}$ This work was supported in part by the U.S. National Institute of Health (R01GM079688, R01GM089866 and R21CA176854), and U.S. National Science Foundation (CBET 0941055 and CBET 1148298). Corresponding author: Xiaoming Liu, liuxm@msu.edu.

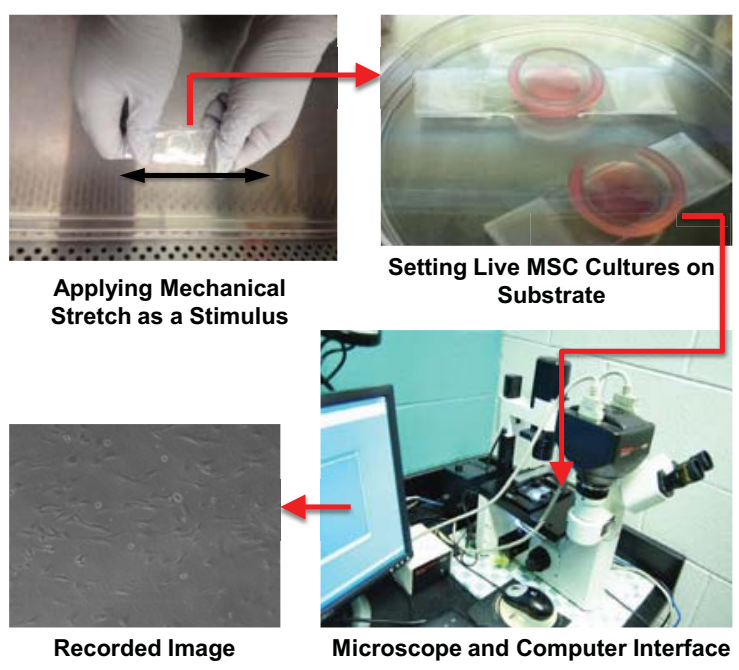

Figure 1: MSC image data collection. Cells are cultured on a substrate with an applied fixed stretch as a stimulus. This substrate is then placed under a microscope for observing cell growth. A computer interfaces with the microscope and captures the images.

(MSCs) have gained enormous interests due to their applications in regenerative medicine. Recent statistics of the Public Clinical Trials database shows the use of MSCs in 206 clinical trials covering a wide range of therapeutic applications [25]. Therefore, studies pertaining to the MSCs behavior are gaining significant importance with time.

The goal of studying MSCs behavior is to understand the cell responses and the mechanism by which the cell shows a particular response to a mechanical stimulus [8]. Specifically, scientists perform MSCs experiments using diverse culturing conditions, capture a large number of images, and analyze them for detecting specific patterns. Manual analysis of these images cannot be effective, therefore commercial tools have been developed to assist researchers. However, these tools are not sufficiently accurate and involve a 


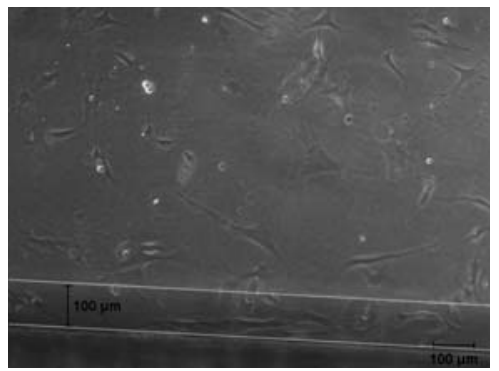

(a) 35PDMS+Cuts

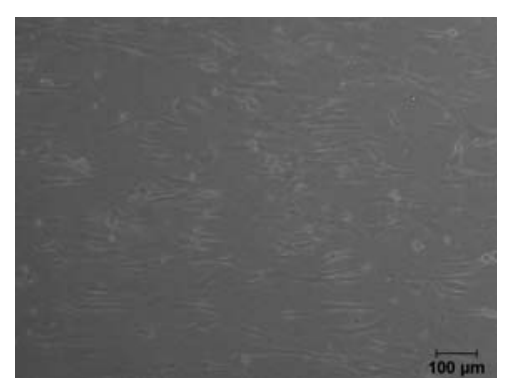

(b) 10PDMS+Stretch

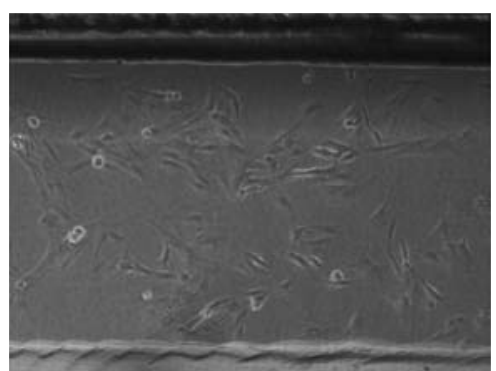

(c) Plastic+Cuts

Figure 2: Example images from each of the three datasets.

lot of manual work. For example, in a recent evaluation of endothelial cell density estimation with three commercial tools, two out of three analysis results were not comparable to the ground truth and significantly overstated the cell density [20]. Since MSCs have a higher differentiating nature than endothelial cells, such analysis can be even more challenging. Hence, there is a strong need for a tool that accurately performs automatic MSCs analysis.

However, due to the diverse nature of MSCs experiments and the special differentiating characteristics of MSCs, even the segmentation of MSCs, the first step in automatic analysis, can be a challenge. To understand the MSCs behavior, we collect datasets of MSCs from three experimental conditions with different mechanical stimuli: (i) 10: 1 crosslinked polydimethylsiloxane (PDMS) substrate kept under mechanical stretch, (ii) 35: 1 crosslinked PDMS substrate with applied cuts, and (iii) plastic substrate with cuts. In this paper, these conditions are denoted as 10PDMS+Stretch, 35PDMS+Cuts, and Plastic+Cuts, respectively. Figure 1 shows our data collection procedure. A number of traditional threshold-based image segmentation approaches fail to achieve satisfying results on our datasets. The best performing approach, the recent binarizationbased extraction of alignment score (BEAS) [27] approach, uses an adaptive threshold based segmentation. Its threshold is estimated based on the mean and standard deviation, which cannot cover sufficient statistics of a cell image distribution. Our analysis shows that different local regions in the cell images with the same mean and standard deviation may have different optimal thresholds. This raises the need of a more effective MSC segmentation approach.

To address this problem, this paper proposes a novel and effective approach to segment MSC images captured under different mechanical stimuli. To deal with the challenge of segmentation on our MSC datasets, we aim to understand the characteristics of our data by using three simple quantitative measures and by analyzing the intensity distributions of local cell patches in relation to the performance of traditional approaches. Furthermore, by significantly extending the BEAS approach, we propose a novel combination of threshold estimation model, as well as more effective fea- tures that relate to the rate of rise in the intensity distribution of a cell image. Comparison of our approach with traditional approaches clearly shows the superiority of our approach on the MSC datasets.

In summary, this paper has three main contributions:

$\diamond$ We propose a novel and effective approach for accurate segmentation of MSC images obtained from live cultures under diverse mechanical stimuli.

$\diamond$ We collect three diverse datasets of MSCs exposed to different mechanical stimuli. To facilitate future research and comparison, our databases are publicly available.

$\diamond$ We conduct the first comparative study of seven well-known image segmentation approaches on these three datasets of MSC images.

\section{Prior Work}

Automatic cell segmentation is not new to our research community. However, it is important to note that the design of cell segmentation algorithms varies significantly depending on factors such as the morphological and behavioral characteristics of different cells, and the objective of the cell study. To the best of our knowledge, there is no comprehensive study in the literature that specifically aims for MSC segmentation. Therefore, this paper focuses on accurate cell segmentation in our challenging MSC datasets. As shown in Fig. 2, we observe significant variations in MSC images when cultured under different experimental setups.

In some studies, florescence is used to highlight the cells in an image, which makes cell segmentation substantially easier. For example, Wiliem et al. use such images to classify human epithelial cells [26]. Studying lymphocytes on such images is the focus of [16]. Similarly, two recent works $[5,11]$ use color-based images for cancer detection. However, in our study the behavior of live cultures of cells has to be observed without using fluorescence or staining.

In the literature, researchers have adopted various approaches to cell segmentation. In $[9,28]$, live cultures of the cells are used to detect mitotic events of cells and track them across frames. A bright spot appears in the image when a cell goes through mitosis. Authors in [28] adopt the level set method, whereas the segmentation technique of [9] is based on a specific microscopy imaging model. On the other 


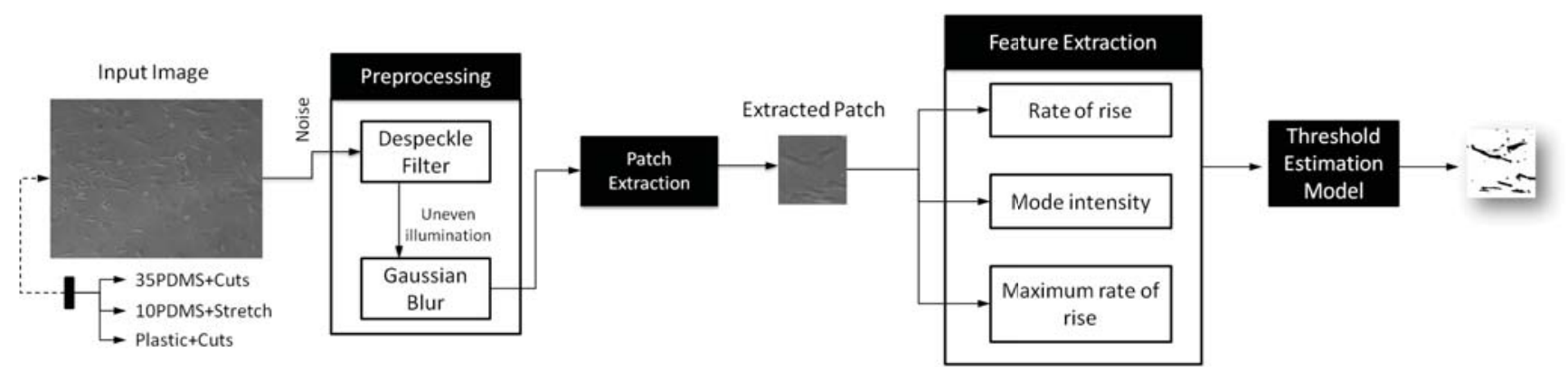

Figure 3: Overall architecture of our approach.

hand, many studies also employ supervised learning-based approaches, such as neural network [15,16] and AAM [14]. Authors in [4] adopt a learning-based template matching approach to segment cell nuclei in microscopy images. However, learning-based approaches might not be suitable for a rapid large scale analysis on cell data, due to the timeconsuming manual labeling of large data and training. Further, approaches that utilize morphological models for cell segmentation, might not be a preferred choice for our MSC datasets due to the highly differentiating nature of MSC, i.e., it continually changes its morphology.

Gradient based approaches are also of interest to many researchers [6,23]. In [6], behaviors of the fibroblast cells are studied and mitotic events are tracked which appeared as bright spots in their images. However, gradient based techniques are not known for performing well on low contrast and noisy images [27]. Further, study in [6] does not explicitly describe the image diversity in its dataset. In contrast, due to substantial diversity in our datasets, we thoroughly study dataset based variations using quantitative measures, while designing our proposed approach.

Thus, the nature of our MSCs study directs us to prefer the use of learning-free approaches that are independent of utilizing any morphological models for cell segmentation, such as those mentioned in $[18,19,22,29]$. In a similar BEAS approach [27], Xu et al. study the cellular orientations and show that adaptive thresholding is effective in segmenting a diverse set of cell images. After applying the preprocessing steps to the cell images, they use the local thresholding approach of Savoula and Pietikäinen [22] for segmentation. They obtain significantly better results in comparison to Fast Fourier Transform-Radial Sum [17] and gradient based approaches [12]. This motivates us to study the BEAS approach and further extend it to tackle our data.

\section{The Proposed Approach}

The overview of our approach is shown in Fig. 3. A cell image of MSC datasets first goes through the preprocessing step, where we use the despeckle filter to reduce the image noise and Gaussian blur to remove the effects of uneven illumination. A similar preprocessing step is adopted in [27]. We use the standard implementation in ImageJ [21], with a standard deviation of 2 for Gaussian blur.

Our analysis shows that there is a high overlap between the cell and background intensities, and diverse intensity distributions at different areas of a cell image. Thus, to reduce the intensity variation in the cell and background pixels, we extract local patches from the original image. These patches are passed through the feature extraction step, after which our threshold estimation model calculates an accurate threshold to segment the MSCs. In the remaining sections, we provide the details of three steps: patch extraction, feature extraction, and threshold estimation.

\subsection{Patch Extraction}

Although after preprocessing our cell images might be less noisy, they are still challenging to segment, as shown by the performance of conventional approaches in Sec. 4. Hence, we perform a comprehensive analysis on our database to understand its characteristics and complexity. Our analysis shows that there are huge variations in the intensities of cell and background pixels, and thus there is a significant overlap between the pixel intensities of cells and background. As a result, using a single and global threshold to segment the entire image might not be an effective approach. To reduce the image complexity, we randomly extract patches of size $300 \times 300$ from the original image. The patch size is chosen in order to capture a reasonable amount of cells with background regions.

We now observe and measure the effect of this patch extraction step. To quantitatively evaluate the reduction of complexity, we define three measures, as shown in Table 1. Higher values of these measures indicate higher complexity for segmentation. Also, these measures help us to compare the complexity of all three datasets and can potentially be used by researchers for comparing their datasets with ours. We now define these measures as follows:

Cell-pixel variance If the pixel intensities of a cell region show a high variance, this can pose a challenge for cell segmentation. If $N_{c}$ is the total number of pixels in all cell regions, $p_{i}^{c}$ represents the intensity of a pixel $i$ within cell regions, and $m\left(p_{i}^{c}\right)$ is the mean intensity of all cell pixels, we calculate the cell-pixel variance as follows, 
Table 1: Quantitative measures for three datasets.

\begin{tabular}{|c|c|c|c|c|}
\cline { 2 - 5 } \multicolumn{1}{c|}{} & Measures & 35PDMS+Cut & 10PDMS+Stretch & Plastic+Cut \\
\hline Original & $s_{c}$ & $3.167 \pm 0.964$ & $5.494 \pm 0.114$ & $7.841 \pm 0.574$ \\
image & $s_{b}$ & $6.372 \pm 0.029$ & $7.088 \pm 0.330$ & $10.389 \pm 0.431$ \\
& $\eta$ & 0.869 & 0.896 & 0.810 \\
\hline Patch & $s_{c}$ & $2.599 \pm 0.547$ & $2.556 \pm 0.853$ & $5.930 \pm 0.182$ \\
& $s_{b}$ & $5.763 \pm 0.537$ & $5.259 \pm 0.678$ & $9.652 \pm 1.619$ \\
& $\eta$ & 0.878 & 0.903 & 0.810 \\
\hline
\end{tabular}

$$
s_{c}=\frac{1}{N_{c}} \sum_{i=1}^{N_{c}}\left(p_{i}^{c}-m\left(p_{i}^{c}\right)\right)^{2} .
$$

Table 1 shows that $s_{c}$ is high for all the original images and reduced significantly for the extracted patches. This means a potential decrease in the overlap of cell and background pixel intensities and an increase in the ease of segmentation. Also, we note that $s_{c}$ is the highest for the Plastic dataset.

Background-pixel variance Similarly, the cell images are difficult to segment if the distribution of background pixels has a large variance. We define this measure as follows,

$$
s_{b}=\frac{1}{N_{b}} \sum_{i=1}^{N_{b}}\left(p_{i}^{b}-m\left(p_{i}^{b}\right)\right)^{2},
$$

where $N_{b}$ is the number of background pixels, $p_{i}^{b}$ is the intensity of a background pixel, and $m\left(p_{i}^{b}\right)$ represents the mean intensity of background pixels. Again, Table 1 shows decrease in $s_{b}$ due to the patch extraction step. However, the overall decrease of $s_{b}$ is less than that of $s_{c}$. Furthermore, we observe the highest $s_{b}$ for patches from the Plastic dataset. Thus, the two measures, $s_{c}$ and $s_{b}$, clearly show that the Plastic dataset is the most challenging, whereas these measures are the lowest for 10PDMS patches.

Cell to background ratio To understand the contrast between the average cell intensity and the average background intensity, we define $\eta=\frac{m\left(p_{i}^{c}\right)}{m\left(p_{i}^{b}\right)}$. In case of no cells, this measure has a zero value. On the other hand, in the most challenging case $\eta=1$, which means the mean cell intensity equals to the mean background intensity. It is desirable to have low values of $\eta$. Higher values mean a very small difference between the intensity levels of cell and background regions in an image. Table 1 shows that all our datasets have $\eta>0.8$, and these high values indicate all our datasets to be challenging. However, no significant change of $\eta$ is observed as a result of patch extraction. This shows that local patches can have smaller intensity variations in both the cell and background region, but not necessary higher contrast in their intensity levels.

\subsection{Distribution Analysis and Feature Extraction}

In order to design an accurate model for segmenting the MSCs in our three datasets, we need to extract and utilize the most effective features from the image data. For this purpose we propose a novel combination of features, listed below, for our threshold estimation model:

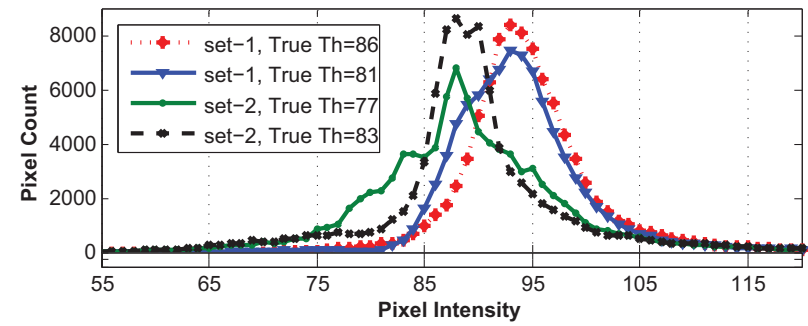

Figure 4: Two sets of image pairs with the same mean and standard deviation but different optimal thresholds (shown as True Th).

$\diamond$ Rate of rise,

$\diamond$ Mode intensity for a patch,

$\diamond$ Maximum rate of rise for a dataset.

It is important to provide the analysis that leads to the design of this feature combination. Thus, rather than merely introducing the features, we explain our two-fold analysis to discover the above feature set: (i) analyze the features in prior threshold-based approaches, and (ii) explore the relevance of these features w.r.t. our datasets.

\subsubsection{Features of Prior Threshold-based Approaches}

Most well-known threshold-based approaches show a bias towards using the mean and standard deviation as the features $[10,18,19,22]$. These approaches have performed very well on some datasets. For example, in [27], the authors design the BEAS approach to segment the diverse sets of cell images and report accurate results. However, when we analyze our datasets using only the mean and standard deviation based measures, we observe the need of more discriminating features. This will be explained in the next section.

\subsubsection{Relevance of Features on Our Datasets}

To analyze the relevance of any features to our dataset, we first study the pixel intensity distributions in our cell images. For a general non-Gaussian distribution, the mean and standard deviation can not completely describe the information of a distribution. Two very different distributions could have the same mean and variance. This is also true for the pixel intensity distribution of cell images. In other words, two cell images with the same mean and standard deviation can have different optimal segmentation thresholds. For example, in Fig. 4, the mean and standard deviations of two distributions in a pair are approximately the same but their optimal thresholds are significantly different.

This can create challenges for many threshold-based approaches including [27] on our datasets. Hence, to find a differentiating feature, we study the common characteristics of all the distributions and particularly of confusing cases such as those in Fig. 4. We find that even for a small patch with a few cells, the pixel intensities of some cells can lie in a very different range than other cells. Similarly not all background regions have the same range of pixel intensities. 
Some background regions can be much darker and their intensities can also overlap with those of cells. We observe the rate of rise as a main factor that can characterize the diverse distributions. The rate of rise is defined as the rate at which the pixel intensity in the histogram of a cell image rises from its low values (mainly cell regions) towards the peak value (mainly background). As shown in Fig. 4, even though the mean and standard deviations are the same, the rates of rise are different for the various distributions, representing the different nature of the cell and background distribution. Therefore, to utilize this feature in our threshold estimation model, we use the power of the exponential distribution to measure the rate of rise,

$$
\mathbf{y}=A e^{\beta \mathbf{x}},
$$

where $\mathbf{x}$ are pixel intensity values in a patch that are less than or equal to the most frequent intensity value and $\mathbf{y}$ is the count of pixels for each intensity value in $\mathbf{x}$. We calculate the rate of rise, $\beta$, using a standard curve fitting procedure based on a pair of $\mathbf{x}$ and $\mathbf{y}$.

Further, for each patch this rise ends as it reaches the peak value presenting the most frequent intensity called "mode". The mode intensity can be different for each patch and hence we consider it as a feature in combination with the rate of rise. The concept of rate of rise is somewhat similar to the kurtosis of a distribution as both tend to measure the peakedness of a distribution but unlike kurtosis, to compute rate of rise, we consider the part of the distribution that lies to the left of the mode intensity. Moreover, our feature set also includes a parameter that estimates the maximum $\beta$ of all patches in a dataset. This feature will be explained in the next section of threshold estimation model.

\subsection{Threshold Estimation Model}

In addition to effective features, we also need a welldesined model to utilize these features for threshold estimation. Considering our analysis and accurate prior results reported by [27], we significantly evolve the BEAS threshold model to a more accurate model. To make this paper selfcontained, we first introduce the BEAS threshold model. As shown in Eqn. 4, the BEAS model calculates a threshold $T$ by an addition of two parts: a reference part and a shift part.

$$
T=m+k m\left(\frac{s}{R}-1\right)
$$

where $m$ is the mean intensity of a patch and $s$ is the standard deviation of a patch. The maximum standard deviation of a dataset, $R$, can be learnt either from the data or provided by the user. The parameter $k$ is specified by the user to tune the model.

Typically the threshold is estimated at a distance from the mean value. Hence, $m$ acts as the reference part and $k m\left(\frac{s}{R}-1\right)$ measures the shift from $m$ towards the estimated threshold. It is important to note that the shift part is a critical term and determines the accuracy of the threshold-based segmentation. As mentioned before, this model is primarily determined by the mean and standard deviation and our experiments show that it is not sufficiently accurate on our datasets. Therefore we extend both the shift and the reference parts to explore the efficacy of our feature set.

Extend the reference part Our approach focuses on the discriminative ability of the shape of the rise in a cell image distribution. This rise ends completely when the pixel intensity equals the mode value and therefore the mode acts as a reference in our model. Denoted as $m_{p}$, the mode of a patch is the most frequent pixel intensity within a patch.

Extend the shift part The shift part can further be seen as a product of two terms, $k m$ and $\left(\frac{s}{R}-1\right)$. To explore and compare the effectiveness of our approach, we first extend the second term and utilize the discriminating ability of the rate of rise $\beta$. The extension can be written as $\left(\frac{\beta}{\ell \beta_{\mu}}-1\right)$, where $\beta$ is the rate of rise for a patch and $\ell \beta_{\mu}$ estimates the maximum rate of rise in one dataset. In our experiments, $\beta_{\mu}$ is calculated by averaging $\beta$ of randomly selected 5 patches from a dataset, and $\ell$ is set to be a constant of 2 .

The first term in the product, $k m$, is extended in our model to $k m_{p}$. We use $k=0.35$ in all our experiments. In our model we further weight this term with $\frac{m_{p}}{255}$, which indicates the position of the mode w.r.t. the range from 0 (darkest) to 255 (brightest).

After incorporating all our extensions, we use the following model to calculate the threshold $T$ of a local patch:

$$
T=m_{p}+\frac{k m_{p}^{2}}{255}\left(\frac{\beta}{\ell \beta_{\mu}}-1\right) .
$$

In summary, $\beta_{\mu}$ is calculated once for each of our three datasets. To estimate a threshold for each patch in a specific dataset, $\beta$ and its mode intensity $m_{p}$ are calculated. Suitable constants for $k$ and $\ell$ are used for our model. In this paper we do not focus on thoroughly finding the optimal values for $\ell$ and $k$. However, it is important to note that our model is less sensitive to the values of $k$ than that of $\ell$.

\section{Experiments and Results}

In this section we test our approach on MSC image patches from three datasets that are collected for analyzing the responses of cells to mechanical stimuli. We first explain our dataset collection process, and then present the detailed experimental results. Finally we provide a brief discussion on the relationship between the accuracy of higher level analysis and the role of cell segmentation.

\subsection{Dataset Collection}

We begin by explaining the details of our dataset and the experimental setup for collecting our data. As the focus of our dataset, MSCs are isolated from Sprague-Dawley rats. Femurs and tibias are dissected from 6-8 week old rat as described in [31]. The cells are grown over a period of 5 days and imaged using the Leica DM IL inverted microscope 
Table 2: Comparison of threshold estimation errors $e_{t}$.

\begin{tabular}{|c|c|c|c|}
\hline Algorithm & 35PDMS+Cuts & 10PDMS+Stretch & Plastic+Cuts \\
\hline Huang & $9.11 \pm 1.88$ & $7.4 \pm 3.02$ & $14.50 \pm 4.16$ \\
\hline Li & $11.44 \pm 2.91$ & $8.72 \pm 2.94$ & $16.89 \pm 5.09$ \\
\hline ME & $31.44 \pm 8.94$ & $24.12 \pm 10.24$ & $37.61 \pm 17.50$ \\
\hline Otsu & $16.11 \pm 9.48$ & $10.16 \pm 4.30$ & $19.39 \pm 6.57$ \\
\hline SH & $33.78 \pm 27.66$ & $14.92 \pm 12.62$ & $49.94 \pm 32.45$ \\
\hline Yen & $32.44 \pm 8.89$ & $24.32 \pm 12.22$ & $39.61 \pm 20.61$ \\
\hline Ours & $1.32 \pm 0.79$ & $1.36 \pm 1.24$ & $1.626 \pm 1.76$ \\
\hline
\end{tabular}

(Bannockburn, IL) with a 10X objective. For our study, these cells are grown on three different substrates subjected to different stimuli. Each stimulus has a different role in affecting the behavior of the MSCs. We list the conditions as follows: (i) $35: 1$ crosslinked PDMS substrate with cuts, (ii) 10: 1 crosslinked PDMS substrate with applied stretch, and (iii) Plastic substrate with cuts.

Our database contains 28 images of the size $1392 \times 1040$, 10 images of the size $1200 \times 1600$, and 15 images of the size $1392 \times 1040$ in the datasets of 35PDMS+Cuts, 10PDMS+Stretch, and Plastic+Cuts respectively. For performance evaluation, we randomly extract a diverse set of 86 patches, with a size of $300 \times 300$, from the 53 images of MSC databases: 50 patches from 10PDMS+Stretch and 18 patches from both 35PDMS+Cuts and Plastic+Cuts. To facilitate future research and performance comparison, these three MSC databases are publicly available ${ }^{1}$.

\subsection{Results and Comparison}

In order to study the performance of conventional approaches on our data and compare to our approach, we select a diverse set of well-known global and local threshold based approaches. To remove any bias in our coding, we use the standard implementation of these approaches (the auto threshold tool and the auto local threshold tool ${ }^{2}$ ) available in ImageJ. All the approaches are tested on the same local patches extracted from three datasets, which have been passed through the preprocessing step mentioned in Sec. 3. Before we present the results, we briefly introduce the baseline approaches to make this paper self-contained.

$\diamond$ Huang [7] The threshold is calculated based on minimizing the measures of the fuzziness. This approach also makes use of the Shanon's Entropy.

$\diamond \mathbf{L i}$ [13] This technique uses the minimum cross entropy for thresholding. The implementation used in this paper is based on its iterative version.

$\diamond$ BEAS [27] This is a local theshold based approach. It considers a limited surrounding region around each pixel and estimates a threshold to segment a particular pixel based on the mean and standard deviation.

$\diamond$ Max Entropy (ME) [10] This technique uses the entropy of the histogram for thresholding. We use the standard

\footnotetext{
${ }^{1}$ http://www.cse.msu.edu/ liuxm/MSC

2 http://fiji.sc/wiki/index.php
}

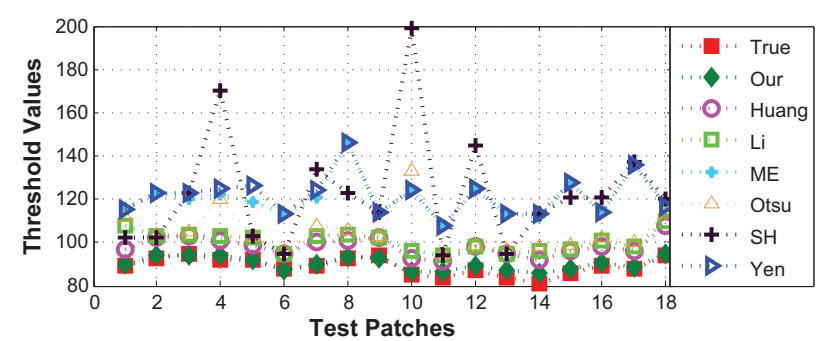

Figure 5: Comparison of estimated thresholds on 35PDMS.

implementation of this approach detailed in [10].

$\diamond$ Otsu [19] This technique is based on minimizing the intra-class variance of the two classes. This intra-class variance is a weighted sum of the variance of two classes.

$\diamond$ ShanBhag (SH) [24] In this entropy-based technique, the image is considered to be composed of two fuzzy sets that correspond to two classes.

$\diamond$ Yen [30] This multi-level thresholding technique is based on two factors: the discrepancy between the original and the thresholded image, and the number of bits required to represent a thresholded image.

To make a comprehensive analysis, we use two different evaluation matrices: (i) the threshold prediction error $e_{t}$, which is the absolute difference between the estimated threshold $T$ and the ground-truth threshold $T_{g}$, and (ii) the similarity score $S$ of the estimated segmentation mask $\mathbf{M}_{g}$ w.r.t. the ground-truth mask M. For each patch in our datasets, we manually adjust the threshold until achieving visually appealing segmentation results. The resultant threshold is treated as the ground-truth threshold $T_{g}$, based on which the resultant segmentation mask is considered as the ground-truth mask $\mathbf{M}_{g}$. We compute these metrics by,

$$
\begin{gathered}
e_{t}=\left|T-T_{g}\right|, \\
S=1-\frac{\| \mathbf{M}-\left.\mathbf{M}_{g}\right|_{2} ^{2}}{N_{M}},
\end{gathered}
$$

where $N_{M}$ is the total number of pixels in the ground-truth mask $\mathbf{M}_{g}$. Now we present our results in three aspects.

Comparing threshold prediction error Other than the BEAS approach, a local threshold method calculating a threshold for each pixel, we expect the other approaches to estimate a threshold value for each testing patch that is closer to the ground-truth threshold $T_{g}$. We show the mean and standard deviation of the threshold prediction error $e_{t}$ in Table 2. Note that the BEAS approach cannot be reported in this table, and its similarity score of the segmentation mask will be presented later. From Table 2, we see that the error is the smallest for our approach in all three datasets while Huang ranks the second. On the other hand, both ME and Yen show least suitability to our datasets. We show the predicted threshold values of all these algorithms on 18 testing patches of 35PDMS+Cuts in Fig. 5. On the same 18 patches, we also perform an unpaired $t$-test for each approach to compare its threshold with the ground truth. We 

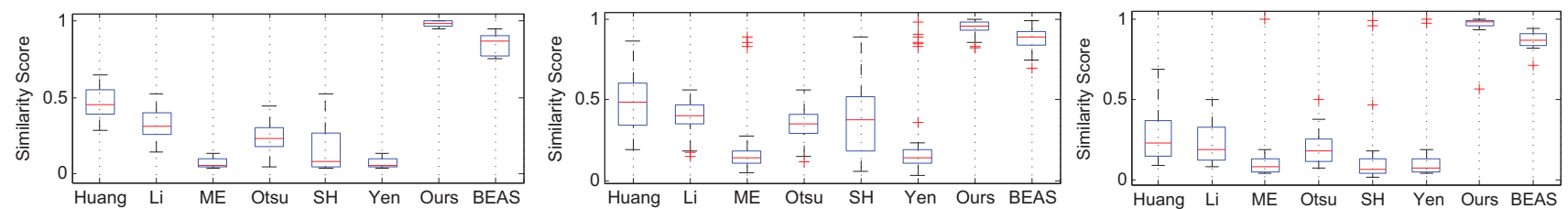

Figure 6: Comparison of similarity scores $S$ on 35PDMS+Cuts (left), 10PDMS+Stretch (middle), and Plastic+Cuts (right).

obtain a t-value of 7.09 for our approach whereas for Huang the $\mathrm{t}$-value is 20.56 , hence showing a higher similarity of our results with the ground truth.

Comparing similarity score In this section, we compare our estimated segmentation mask to the ground-truth mask, since this is a more direct evaluation of the segmenation accuracy than the threshold prediction error. The comparison of similarity scores $S$ in Fig. 6 shows that the resultant masks obtained from our approach are most similar to the ground truth. Considering a confidence interval of $95 \%$, the mean similarity scores of our approach on 35PDMS+cuts, 10PDMS+stretch, and Plastic+cuts are $0.98 \pm 0.008,0.95 \pm$ 0.013 , and $0.95 \pm 0.046$ respectively, whereas the BEAS approach ranks the second with the respective mean similarity scores of $0.85 \pm 0.032,0.88 \pm 0.016$, and $0.86 \pm 0.030$ for the aforementioned three datasets. Furthermore, our method appears to have the minimal variation in similarity scores among all methods, which is a favorable property well reflecting the stability of our segmentation algorithm on diverse cell images. Figure 7 shows examples of the segmentation mask obtained from the best two algorithms.

Comparing results of datasets It is important to see how all the algorithms perform on different datasets. We can relate this study to the discussion on the characteristics of the image data presented in Sec. 3.1 and thus develop insights regarding the overall performance of algorithms. Table 2 shows high errors for all the algorithms on the Plastic dataset, while the errors are mostly the lowest for 10PDMS. Similarly the average value of similarity scores for all the results is the highest for 10PDMS with a value of 0.484 , whereas this value is the lowest for Plastic with 0.379 . This can be related to the fact that the Plastic data also has the highest values for both cell-pixel variance and backgroundpixel variance, as shown in Table 1. On the other hand, these variances are the lowest for the 10PDMS dataset, which is also consistent with the fact that the threshold prediction errors of 10PDMS are the lowest.

\subsection{High Level Analysis and Segmentation}

High level analysis of cell images, such as orientation or density based studies, can be significantly affected by the accuracy of image segmentation results. Even slightly better segmentation performance of one technique over the other can provide very different results in high level analysis. Hence, given our accurate segmentation, we consider our approach to be an enabling tool for high level cell anal- ysis. To elucidate our point, the $4^{\text {th }}$ row of Fig. 7 clearly shows that some unwanted dark regions are obtained with the BEAS approach, which are hard to be removed by post processing. This can happen whenever a method does not perform well in cell segmentation. If automatic density based study or orientation based analysis is performed on such masks, the results of such studies can be significantly erroneous. Therefore, for a truthful high level analysis, it is imperative to first achieve highly accurate cell segmentation, as demonstrated in our proposed approach.

\section{Conclusion and Future Work}

It is commonly believed that the success of high level cell analysis heavily depends on the accuracy of cell segmentation. Therefore, this paper aims to design an accurate cell segmentation model for the challenging images obtained under diverse experimental conditions. We evaluate the efficacy of our approach on three MSC image datasets obtained under the influence of three different sets of culture surface and mechanical stimuli. Comparison with previous methods shows the superiority of our approach. Our approach is the outcome of a thorough analysis of the different sets of MSC images and the discovery of the discriminative feature such as the rate of rise of the intensity distribution.

One next step is to fuse the patch-based segmentation into a segmentation mask for an entire cell image. Also we will utilize our accurate segmentation to design an automatic tool for high level cell analysis, such as studying the distribution of cell orientation in response to different mechanical stimuli. Furthermore, the generalization of our approach to other applications is also our future goal.

\section{References}

[1] http://minorityhealth.hhs.gov/templates/brows e.aspx?lvl=3\&lvlid=555.

[2] http://report.nih.gov/NIHfactsheets/ViewFactS heet.aspx?csid=62\&key=R\#R.

[3] G. Abouna. Organ shortage crisis: problems and possible solutions In Transplantation Proceedings, volume 40, pages 34-38. Elsevier, 2008.

[4] C. Chen, W. Wang, J. A. Ozolek, and G. K. Rohde. A flexible and robust approach for segmenting cell nuclei from 2D microscopy images using supervised learning and template matching. Cytometry Part A, 83(5):495-507, 2013.

[5] Y. M. George, B. M. Bagoury, H. H. Zayed, and M. I. Roushdy. Automated cell nuclei segmentation for breast fine needle aspiration cytology. Signal Processing, 93(10):2804-2816, 2013.

[6] D. House, M. L. Walker, Z. Wu, J. Y. Wong, and M. Betke. Tracking of cell populations to understand their spatio-temporal behavior in 

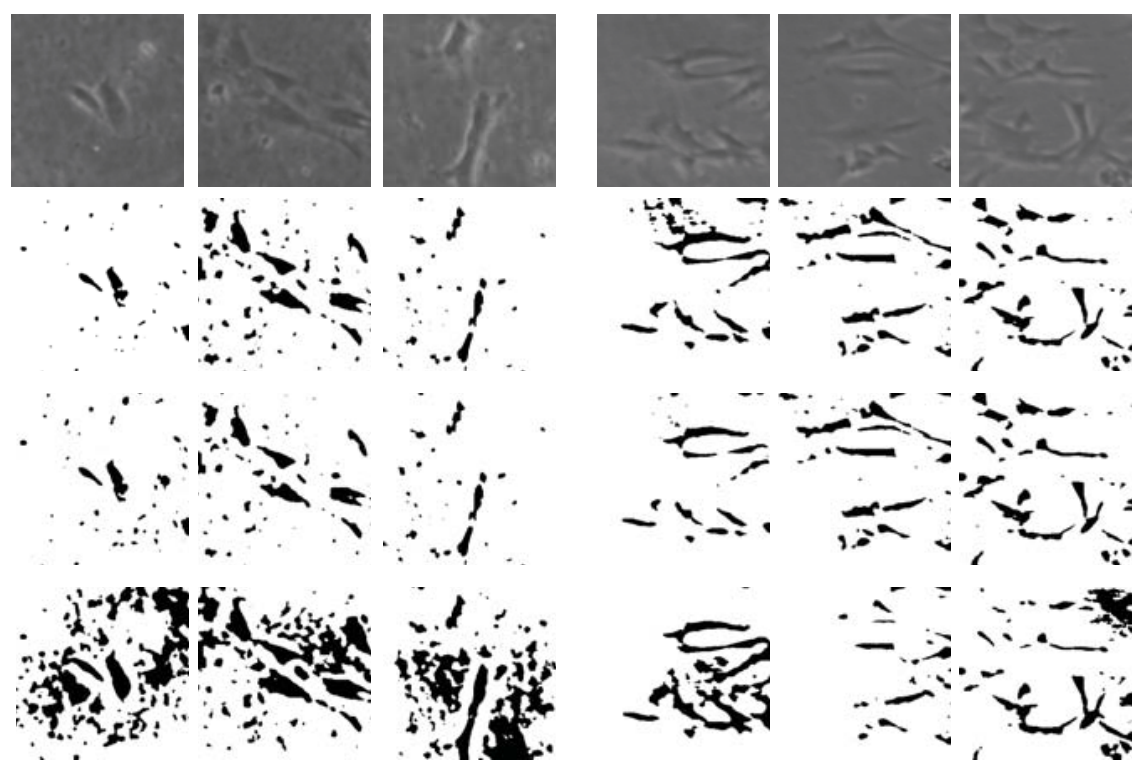

(b) 10PDMS+Stretch
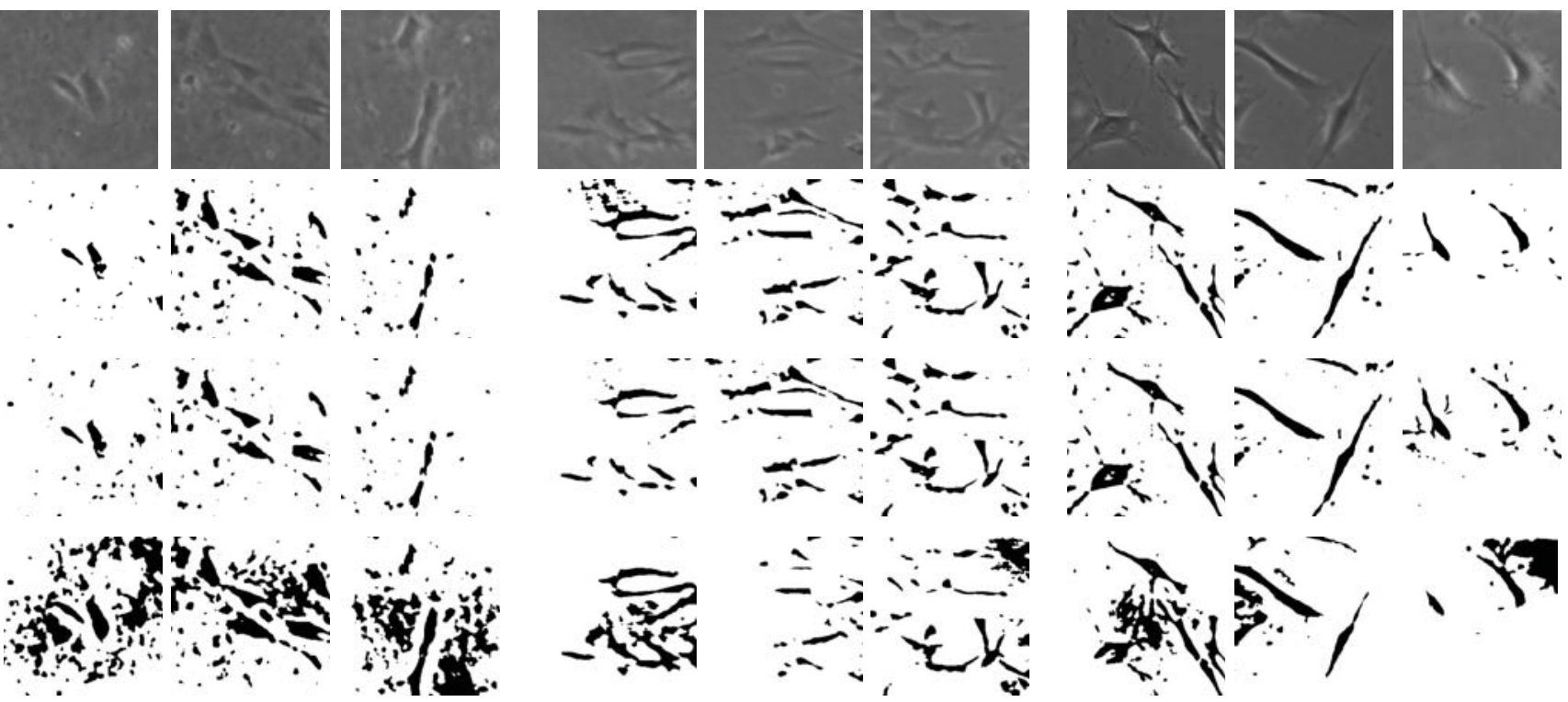

(a) 35PDMS+Cuts

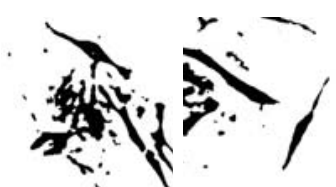

(c) Plastic+Cuts

Figure 7: Examplar cell segmentation results (three examples per dataset) with the original image, the ground-truth mask, our approach, and the BEAS approach, vertically from the top row to the bottom row. Note the similarity of our approach to the ground truth and the superiority of our approach over the BEAS approach.

response to physical stimuli. In CVPR Workshops, pages 186-193, 2009.

[7] L.-K. Huang and M.-J. J. Wang. Image thresholding by minimizing the measures of fuzziness. Elsevier-PR, 28(1):41-51, 1995.

[8] P. A. Janmey and C. A. McCulloch. Cell mechanics: integrating cell responses to mechanical stimuli. Annu. Rev. Biomed. Eng., 9:1-34, 2007.

[9] T. Kanade, Z. Yin, R. Bise, S. Huh, S. Eom, M. F. Sandbothe, and M. Chen. Cell image analysis: Algorithms, system and applications. In WACV workshop, pages 374-381, 2011.

[10] J. Kapur, P. K. Sahoo, and A. Wong. A new method for gray-level picture thresholding using the entropy of the histogram. Computer Vision, Graphics, and Image Processing, 29(3):273-285, 1985.

[11] S. S. Kecheril, D. Venkataraman, J. Suganthi, and K. Sujathan. Automated lung cancer detection by the analysis of glandular cells in sputum cytology images using scale space features. Signal, Image and Video Processing, pages 1-13, 2013.

[12] A. A. Lee, D. A. Graham, C. S. Dela, A. Ratcliffe, and W. J. Karlon. Fluid shear stress-induced alignment of cultured vascular smooth muscle cells. J. Biomech Engineering, 124(1):37-43, 2002.

[13] C. Li and P. K.-S. Tam. An iterative algorithm for minimum cross entropy thresholding. Elsevier-PRL, 19(8):771-776, 1998.

[14] X. Liu, P. Annangi, M. Gupta, B. Yu, D. Padfield, J. Banerjee, and K. Krishnan. Learning-based scan plane identification from fetal head ultrasound images. In Proc. SPIE, Medical Imaging, volume 8320,2012

[15] K. Nandy, P. R. Gudla, R. Amundsen, K. J. Meaburn, T. Misteli, and S. J. Lockett. Automatic segmentation and supervised learningbased selection of nuclei in cancer tissue images. Cytometry Part A, 81(9):743-754, 2012

[16] T. W. Nattkemper, H. J. Ritter, and W. Schubert. A neural classifier enabling high-throughput topological analysis of lymphocytes in tissue sections. IEEE T-ITB, 5(2):138-149, 2001.

[17] C. P. Ng, B. Hinz, and M. A. Swartz. Interstitial fluid flow induces myofibroblast differentiation and collagen alignment in vitro. J. of cell science, 118(20):4731-4739, 2005.

[18] W. Niblack. An introduction to digital image processing. PrenticeHall, 1986.
[19] N. Otsu. A threshold selection method from gray-level histograms. IEEE T-SMC, 9(1):62-66, 1979.

[20] M. O. Price, K. M. Fairchild, and F. W. Price Jr. Comparison of manual and automated endothelial cell density analysis in normal eyes and DSEK eyes. Cornea, 32(5):567-573, 2013

[21] W. Rasband. Imagej. http://rsbweb. nih. gov/ij/, 2008.

[22] J. Sauvola and M. Pietikäinen. Adaptive document image binarization. Elsevier-PR, 33(2):225-236, 2000

[23] I. Seroussi, D. Veikherman, N. Ofer, S. Yehudai-resheff, and K. Keren. Segmentation and tracking of live cells in phase-contrast images using directional gradient vector flow for snakes. J. of $\mathrm{Mi}$ croscopy, 247(2):137-146, 2012.

[24] A. G. Shanbhag. Utilization of information measure as a means of image thresholding. Elsevier-CVGIP, 56(5):414-419, 1994.

[25] S. Wang, X. Qu, and R. C. Zhao. Clinical applications of mesenchymal stem cells. J. Hematol. Oncol., 5(19):1-9, 2012

[26] A. Wiliem, Y. Wong, C. Sanderson, P. Hobson, S. Chen, and B. C. Lovell. Classification of human epithelial type 2 cell indirect immunofluoresence images via codebook based descriptors. WACV, pages 95-102, 2013.

[27] F. Xu, T. Beyazoglu, E. Hefner, U. A. Gurkan, and U. Demirci. Automated and adaptable quantification of cellular alignment from microscopic images for tissue engineering applications. Tissue Engineering Part C: Methods, 17(6):641-649, 2011.

[28] F. Yang, M. A. Mackey, F. Ianzini, G. Gallardo, and M. Sonka. Cell segmentation, tracking, and mitosis detection using temporal context. In MICCAI, pages 302-309. 2005

[29] F. Yang, M. A. Mackey, F. Ianzini, G. M. Gallardo, and M. Sonka. Segmentation and quantitative analysis of the living tumor cells using large-scale digital cell analysis system. In Proc. SPIE, Medical Imaging, volume 5370, pages 1755-1763, 2004.

[30] J.-C. Yen, F.-J. Chang, and S. Chang. A new criterion for automatic multilevel thresholding. IEEE T-IP, 4(3):370-378, 1995.

[31] L. Zhang, L. C. Seitz, A. M. Abramczyk, L. Liu, and C. Chan camp initiates early phase neuron-like morphology changes and late phase neural differentiation in mesenchymal stem cells. Cellular and Molecular Life Sciences, 68(5):863-876, 2011. 